\title{
Bayes approach to study scale parameter of log logistic distribution
}

\author{
Wajiha Nasir ${ }^{1 *}$, Maria ${ }^{2}$, Muhammad Aslam ${ }^{3}$ \\ ${ }^{1},{ }^{3}$ Department of Basic Sciences, Riphah International University Islamabad, Pakistan \\ 2 Department of Statistics, University of Sargodha, Pakistan \\ *Corresponding author E-mail: wajiha.nasir19@gmail.com
}

Copyright (C)2015 Wajiha Nasir et. all. This is an open access article distributed under the Creative Commons Attribution License, which permits unrestricted use, distribution, and reproduction in any medium, provided the original work is properly cited.

\begin{abstract}
Scale parameter of Log logistic distribution has been studied using Bayesian approach. Posterior distribution has derived by using non informative prior. Posterior distribution is not in close form so we have work with quadrature numerical integration. Various loss functions has been utilized to derive the Bayes estimators and their corresponding risks. Simulation study has been performed to compare the performance of different estimators.
\end{abstract}

Keywords: Informative Prior, Bayes Estimates, Posterior Risks, Loss Functions, Monte Carlo simulation.

\section{Introduction}

Log logistic distribution with two parameters i.e. scale and shape, which is a real life distribution and use in different fields of life, also known as Fisk distribution. [1] has obtained three parameter Log Logistic distribution by introducing a new method of estimation. [2] has used Log Logistic model for the study of Dose response in weed science.

[3] has used Log Gumbel and Log Logistic distribution in hydrological analysis

[4] has used maximize ratio test to differentiate among Weibull distribution and Log Logistic distribution.[5] has used Bayesian approach for Log Logistic distribution to check the reliability of distribution and also has derived the Posterior distributions by using weakly informative priors. [6] have used the method of maximum likelihood estimation to estimate the parameters of Log Logistic distribution and also by using the EM algorithm based on progressive type-II right censored sample.

The p.d.f for Log Logistic distribution is

$f(x ; \alpha, \beta)=\frac{\alpha x^{\alpha-1}}{\beta^{\alpha}\left[1+\left(\frac{x}{\beta}\right)^{\alpha}\right]^{2}}$ where $\alpha, \beta>0$

where $\alpha$ is scale parameter and $\beta$ is shape parameter.

We will study Bayes estimators of scale parameter with known shape parameter of Log Logistic distribution . We will use four loss functions using uniform and Jeffery priors. 


\section{Posterior Distribution}

Posterior distribution is derived by using uniform and Jeffery prior.

\subsection{Posterior Distribution using Uniform prior}

Uniform prior as non informative prior with parameter $\alpha$ is

$p(\alpha) \propto 1 \quad 0<\alpha<\infty$

The posterior distribution of scale parameter for given data using $\left(x=x_{1}, x_{2}, \ldots, x_{n}\right)$ is:

$p(\alpha \mid \mathrm{x})=\frac{\alpha^{n}}{u_{1}} e^{\alpha \ln \sum_{i=1}^{n} x_{i}-2 \ln \sum_{i=1}^{n}\left[1+\left(\frac{x_{i}}{\beta}\right)^{\alpha}\right]-n \alpha \ln \beta}$

where

$u_{1}=\int_{0}^{\infty} \alpha^{n} e^{\alpha \ln \sum_{i=1}^{n} x_{i}-2 \ln \sum_{i=1}^{n}\left[1+\left(\frac{x_{i}}{\beta}\right)^{\alpha}\right]-n \alpha \ln \beta} d \alpha$

The posterior distribution is not in close form, so we will solve it numerically.

Jeffreys prior as non informative prior with parameter $\alpha$ is

$p(\alpha) \propto \frac{1}{\alpha} \quad 0<\alpha<\infty$

The posterior distribution of scale parameter for given data using $\left(x=x_{1}, x_{2}, \ldots, x_{n}\right)$ is:

$p(\alpha \mid \mathrm{x})=\frac{\alpha^{n-1}}{u_{2}} e^{\alpha \ln \sum_{i=1}^{n} x_{i}-2 \ln \sum_{i=1}^{n}\left[1+\left(\frac{x_{i}}{\beta}\right)^{\alpha}\right]-n \alpha \ln \beta}$

where

$u_{2}=\int_{0}^{\infty} \alpha^{n-1} e^{\alpha \ln \sum_{i=1}^{n} x_{i}-2 \ln \sum_{i=1}^{n}\left[1+\left(\frac{x_{i}}{\beta}\right)^{\alpha}\right]-n \alpha \ln \beta} d \alpha$

The posterior distribution is not in close form, so we will solve numerically.

\section{Bayes Estimators and respective Corresponding Risks}

Square error, precautionary, simple precautionary and weighted loss functions are utilized to derive the Bayes estimators and their corresponding Bayes posterior risks. Their Bayes estimators and their corresponding Bayes posterior risks using uniform prior are

$\alpha_{1 S E L F}^{*}=\int_{0}^{\infty} \frac{\alpha^{n+1}}{u_{1}} e^{\alpha \ln \sum_{i=1}^{n} x_{i}-2 \ln \sum_{i=1}^{n}\left[1+\left(\frac{x_{i}}{\beta}\right)^{\alpha}\right]-n \alpha \ln \beta} d \alpha$

$\rho_{\left(\alpha_{1 S E L F}^{*}\right)}=\int_{0}^{\infty} \frac{\alpha^{n+2}}{u_{1}} e^{\alpha \ln \sum_{i=1}^{n} x_{i}-2 \ln \sum_{i=1}^{n}\left[1+\left(\frac{x_{i}}{\beta}\right)^{\alpha}\right]-n \alpha \ln \beta} d \alpha-\left(\int_{0}^{\infty} \frac{\alpha^{n+1}}{u_{1}} e^{\alpha \ln \sum_{i=1}^{n} x_{i}-2 \ln \sum_{i=1}^{n}\left[1+\left(\frac{x_{i}}{\beta}\right)^{\alpha}\right]-n \alpha \ln \beta} d \alpha\right)^{2}$

Bayes estimator and Bayes posterior risks under PLF are

$\alpha_{1 P L F}^{*}=\sqrt{\int_{0}^{\infty} \frac{\alpha^{n+2}}{u_{1}} e^{\alpha \ln \sum_{i=1}^{n} x_{i}-2 \ln \sum_{i=1}^{n}\left[1+\left(\frac{x_{i}}{\beta}\right)^{\alpha}\right]-n \alpha \ln \beta} d \alpha}$ 


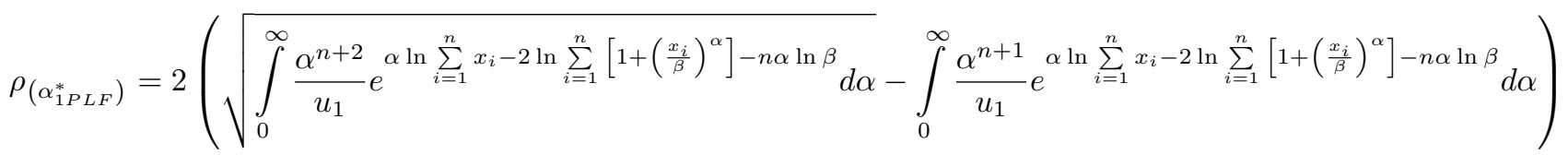

Bayes estimator and Bayes posterior risks under SPLF are

$\alpha_{1 S P L F}^{*}=\sqrt{\frac{\int_{0}^{\infty} \frac{\alpha^{n+1}}{u_{1}} e^{\alpha \ln \sum_{i=1}^{n} x_{i}-2 \ln \sum_{i=1}^{n}\left[1+\left(\frac{x_{i}}{\beta}\right)^{\alpha}\right]-n \alpha \ln \beta} d \alpha}{\int_{0}^{\infty} \frac{\alpha^{n-1}}{u_{1}} e^{\alpha \ln \sum_{i=1}^{n} x_{i}-2 \ln \sum_{i=1}^{n}\left[1+\left(\frac{x_{i}}{\beta}\right)^{\alpha}\right]-n \alpha \ln \beta} d \alpha}}$

$\rho_{\left(\alpha_{1 S P L F}^{*}\right)}=2\left(\sqrt{\left.\int_{0}^{\infty} \frac{\alpha^{n+1}}{u_{1}} e^{\alpha \ln \sum_{i=1}^{n} x_{i}-2 \ln \sum_{i=1}^{n}\left[1+\left(\frac{x_{i}}{\beta}\right)^{\alpha}\right]-n \alpha \ln \beta} d \alpha * \int_{0}^{\infty} \frac{\alpha^{n-1}}{u_{1}} e^{\alpha \ln \sum_{i=1}^{n} x_{i}-2 \ln \sum_{i=1}^{n}\left[1+\left(\frac{x_{i}}{\beta}\right)^{\alpha}\right]-n \alpha \ln \beta} d \alpha-1\right)}\right.$

Bayes estimator and Bayes posterior risks under WLF are

$$
\begin{aligned}
& \alpha_{1 W L F}^{*}= \frac{1}{\int_{0}^{\infty} \frac{\alpha^{n-1}}{u_{1}} e^{\alpha \ln \sum_{i=1}^{n} x_{i}-2 \ln \sum_{i=1}^{n}\left[1+\left(\frac{x_{i}}{\beta}\right)^{\alpha}\right]-n \alpha \ln \beta} d \alpha} \\
& \rho_{\left(\alpha_{1 W L F}^{*}\right)}=\int_{0}^{\infty} \frac{\alpha^{n+1}}{u_{1}} e^{\alpha \ln \sum_{i=1}^{n} x_{i}-2 \ln \sum_{i=1}^{n}\left[1+\left(\frac{x_{i}}{\beta}\right)^{\alpha}\right]-n \alpha \ln \beta} d \alpha-\frac{1}{\int_{0}^{\infty} \frac{\alpha^{n-1}}{u_{1}} e^{\alpha \ln \sum_{i=1}^{n} x_{i}-2 \ln \sum_{i=1}^{n}\left[1+\left(\frac{x_{i}}{\beta}\right)^{\alpha}\right]-n \alpha \ln \beta} d \alpha}
\end{aligned}
$$

Bayes estimators and their corresponding Bayes risks using Jeffreys prior using SELF are

$$
\begin{aligned}
& \alpha_{2 S E L F}^{*}=\int_{0}^{\infty} \frac{\alpha^{n}}{u_{2}} e^{\alpha \ln \sum_{i=1}^{n} x_{i}-2 \ln \sum_{i=1}^{n}\left[1+\left(\frac{x_{i}}{\beta}\right)^{\alpha}\right]-n \alpha \ln \beta} d \alpha \\
& \rho_{\left(\alpha_{2 S E L F}^{*}\right)}=\int_{0}^{\infty} \frac{\alpha^{n+1}}{u_{2}} e^{\alpha \ln \sum_{i=1}^{n} x_{i}-2 \ln \sum_{i=1}^{n}\left[1+\left(\frac{x_{i}}{\beta}\right)^{\alpha}\right]-n \alpha \ln \beta} d \alpha-\left(\int_{0}^{\infty} \frac{\alpha^{n}}{u_{2}} e^{\alpha \ln \sum_{i=1}^{n} x_{i}-2 \ln \sum_{i=1}^{n}\left[1+\left(\frac{x_{i}}{\beta}\right)^{\alpha}\right]-n \alpha \ln \beta} d \alpha\right)^{2}
\end{aligned}
$$

Bayes estimators and their corresponding Bayes risks using PLF are

$$
\begin{aligned}
& \alpha_{2 P L F}^{*}=\sqrt{\int_{0}^{\infty} \frac{\alpha^{n+1}}{u_{2}} e^{\alpha \ln \sum_{i=1}^{n} x_{i}-2 \ln \sum_{i=1}^{n}\left[1+\left(\frac{x_{i}}{\beta}\right)^{\alpha}\right]-n \alpha \ln \beta} d \alpha} \\
& \rho_{\left(\alpha_{2 P L F}^{*}\right)}=2\left(\sqrt{\left.\int_{0}^{\infty} \frac{\alpha^{n+1}}{u_{2}} e^{\alpha \ln \sum_{i=1}^{n} x_{i}-2 \ln \sum_{i=1}^{n}\left[1+\left(\frac{x_{i}}{\beta}\right)^{\alpha}\right]-n \alpha \ln \beta} d \alpha-\int_{0}^{\infty} \frac{\alpha^{n}}{u_{2}} e^{\alpha \ln \sum_{i=1}^{n} x_{i}-2 \ln \sum_{i=1}^{n}\left[1+\left(\frac{x_{i}}{\beta}\right)^{\alpha}\right]-n \alpha \ln \beta} d \alpha\right)}\right.
\end{aligned}
$$


Bayes estimators and their corresponding Bayes risks using SPLF are

$\alpha_{2 S P L F}^{*}=\sqrt{\frac{\int_{0}^{\infty} \frac{\alpha^{n}}{u_{2}} e^{\alpha \ln \sum_{i=1}^{n} x_{i}-2 \ln \sum_{i=1}^{n}\left[1+\left(\frac{x_{i}}{\beta}\right)^{\alpha}\right]-n \alpha \ln \beta} d \alpha}{\int_{0}^{\infty} \frac{\alpha^{n-2}}{u_{2}} e^{\alpha \ln \sum_{i=1}^{n} x_{i}-2 \ln \sum_{i=1}^{n}\left[1+\left(\frac{x_{i}}{\beta}\right)^{\alpha}\right]-n \alpha \ln \beta} d \alpha}}$

$\rho_{\left(\alpha_{2 S P L F}^{*}\right)}=2\left(\sqrt{\left.\int_{0}^{\infty} \frac{\alpha^{n}}{u_{2}} e^{\alpha \ln \sum_{i=1}^{n} x_{i}-2 \ln \sum_{i=1}^{n}\left[1+\left(\frac{x_{i}}{\beta}\right)^{\alpha}\right]-n \alpha \ln \beta} d \alpha * \int_{0}^{\infty} \frac{\alpha^{n-2}}{u_{2}} e^{\alpha \ln \sum_{i=1}^{n} x_{i}-2 \ln \sum_{i=1}^{n}\left[1+\left(\frac{x_{i}}{\beta}\right)^{\alpha}\right]-n \alpha \ln \beta} d \alpha-1\right)}\right.$

Bayes estimators and their corresponding Bayes risks using WLF are

$$
\begin{aligned}
& \alpha_{2 W L F}^{*}= \frac{1}{\int_{0}^{\infty} \frac{\alpha^{n-2}}{u_{2}} e^{\alpha \ln \sum_{i=1}^{n} x_{i}-2 \ln \sum_{i=1}^{n}\left[1+\left(\frac{x_{i}}{\beta}\right)^{\alpha}\right]-n \alpha \ln \beta} d \alpha} \\
& \rho_{\left(\alpha_{2 W L F}^{*}\right)}=\int_{0}^{\infty} \frac{\alpha^{n}}{u_{2}} e^{\alpha \ln \sum_{i=1}^{n} x_{i}-2 \ln \sum_{i=1}^{n}\left[1+\left(\frac{x_{i}}{\beta}\right)^{\alpha}\right]-n \alpha \ln \beta} d \alpha-\frac{1}{\int_{0}^{\infty} \frac{\alpha^{n-2}}{u_{2}} e^{\alpha \ln \sum_{i=1}^{n} x_{i}-2 \ln \sum_{i=1}^{n}\left[1+\left(\frac{x_{i}}{\beta}\right)^{\alpha}\right]-n \alpha \ln \beta} d \alpha}
\end{aligned}
$$

\section{Simulation Study}

We use simulation to check the performance of priors and Loss functions. For this purpose we use different values of scale parameter $\alpha$ by using different sample sizes. We use Mathematica 10 package to generate random number of size $n=\{30,50,70,100\}$ from Log Logistic distribution by using $\alpha \in\{2,4\}$ and $\alpha \in\{5,7\}$. The Bayes posterior risk are in parenthesis. The results for simulation study are summarized in the following tables.

Table 1: Simulation of Bayes estimators and posterior risks under SELF using different Priors

\begin{tabular}{|c|l|l|l|l|l|l|l|l|}
\hline Prior & \multicolumn{5}{|c|}{$\alpha=2, \beta=5$} & \multicolumn{4}{c|}{$\alpha=2, \beta=7$} \\
\hline $\mathrm{n}$ & 30 & 50 & 70 & 100 & 30 & 50 & 70 & 100 \\
\hline UP & 2.1114 & 2.0383 & 2.0349 & 2.0284 & 2.0365 & 2.0295 & 2.0137 & 2.0058 \\
& $(0.1038)$ & $(0.0581)$ & $(0.0413)$ & $(0.0287)$ & $(0.0961)$ & $(0.0505)$ & $(0.0224)$ & $(0.0105)$ \\
\hline JP & 2.0447 & 2.0188 & 2.0113 & 2.0117 & 2.0617 & 2.0401 & 2.0190 & 2.0138 \\
& $(0.1000)$ & $(0.0578)$ & $(0.0408)$ & $(0.0284)$ & $(0.1019)$ & $(0.0590)$ & $(0.0411)$ & $(0.0285)$ \\
\hline & \multicolumn{6}{|c|}{$\alpha=4, \beta=5$} \\
\hline UP & 4.1848 & 4.1097 & 4.0971 & 4.0041 & 4.1564 & 4.0928 & 4.0707 & 3.9935 \\
& $(0.4087)$ & $(0.2362)$ & $(0.1673)$ & $(0.0961)$ & $(0.4022)$ & $(0.2337)$ & $(0.1571)$ & $(0.1588)$ \\
\hline JP & 4.0526 & 4.0546 & 4.0571 & 3.9808 & 4.0970 & 4.0455 & 4.0455 & 3.9235 \\
& $(0.3918)$ & $(0.2333)$ & $(0.1660)$ & $(0.0974)$ & $(0.4008)$ & $(0.2322)$ & $(0.1581)$ & $(0.1561)$ \\
\hline
\end{tabular}


Table 2: Simulation of Bayes estimators and posterior risks under PLF using different Priors

\begin{tabular}{|c|l|l|l|l|l|l|l|l|}
\hline Prior & \multicolumn{5}{|c|}{$\alpha=2, \beta=5$} & \multicolumn{4}{c|}{$\alpha=2, \beta=7$} \\
\hline $\mathrm{n}$ & 30 & 50 & 70 & 100 & 30 & 50 & 70 & 100 \\
\hline UP & 2.1409 & 2.0835 & 2.0469 & 2.0302 & 2.1128 & 2.0669 & 2.0450 & 2.0399 \\
& $(0.0478)$ & $(0.0283)$ & $(0.0200)$ & $(0.0140)$ & $(0.0472)$ & $(0.02815)$ & $(0.02007)$ & $(0.01407)$ \\
\hline JP & 2.0607 & 2.0399 & 2.0255 & 2.0207 & 2.0961 & 2.0450 & 2.0311 & 2.0243 \\
& $(0.0472)$ & $(0.0282)$ & $(0.0201)$ & $(0.0140)$ & $(0.0480)$ & $(0.0283)$ & $(0.0201)$ & $(0.0141)$ \\
\hline & \multicolumn{6}{|c|}{$\alpha=4, \beta=5$} & \multicolumn{6}{c|}{$\alpha=4, \beta=7$} \\
\hline UP & 4.2496 & 4.1513 & 4.1076 & 4.0149 & 4.2646 & 4.1412 & 4.0817 & 4.0324 \\
& $(0.4087)$ & $(0.0566)$ & $(0.0401)$ & $(0.0235)$ & $(0.0951)$ & $(0.0564)$ & $(0.0384)$ & $(0.0413)$ \\
\hline JP & 4.1408 & 4.0982 & 4.0406 & 3.9761 & 4.1511 & 4.1074 & 4.0613 & 3.9552 \\
& $(0.0950)$ & $(0.0567)$ & $(0.0400)$ & $(0.0240)$ & $(0.0952)$ & $(0.0568)$ & $(0.0386)$ & $(0.03854)$ \\
\hline
\end{tabular}

Table 3: Simulation of Bayes estimators and posterior risks under SPLF using different Priors

\begin{tabular}{|c|l|l|l|l|l|l|l|l|}
\hline Prior & \multicolumn{5}{|c|}{$\alpha=2, \beta=5$} & \multicolumn{4}{c|}{$\alpha=2, \beta=7$} \\
\hline $\mathrm{n}$ & 30 & 50 & 70 & 100 & 30 & 50 & 70 & 100 \\
\hline UP & 2.0769 & 2.0438 & 2.0211 & 2.0128 & 2.0809 & 2.0539 & 2.0319 & 2.0230 \\
& $(0.0232)$ & $(0.0139)$ & $(0.0099)$ & $(0.0069)$ & $(0.0232)$ & $(0.0139)$ & $(0.0099)$ & $(0.0069)$ \\
\hline JP & 2.7095 & 2.6077 & 2.5077 & 2.6120 & 2.6958 & 2.7013 & 2.7024 & 2.7033 \\
& $(0.1314)$ & $(0.5522)$ & $(0.1405)$ & $(0.9557)$ & $(0.0024)$ & $(0.8377)$ & $(0.8047)$ & $(0.8111)$ \\
\hline & \multicolumn{6}{|c|}{$\alpha=4, \beta=5$} \\
\hline UP & 4.1978 & 4.0649 & 4.0470 & 3.9856 & 4.1180 & 4.0750 & 4.0334 & 3.9766 \\
& $(0.0232)$ & $(0.0139)$ & $(0.0099)$ & $(0.0060)$ & $(0.0232)$ & $(0.0137)$ & $(0.0095)$ & $(0.0090)$ \\
\hline JP & 4.4902 & 4.4968 & 4.4961 & 4.5019 & 4.4919 & 4.4962 & 4.4975 & 4.5039 \\
& $(0.0203)$ & $(0.4411)$ & $(0.0394)$ & $(0.8446)$ & $(0.0332)$ & $(0.4717)$ & $(0.3028)$ & $(0.8111)$ \\
\hline
\end{tabular}

Table 4: Simulation of Bayes estimators and posterior risks under WLF using different Priors

\begin{tabular}{|c|l|l|l|l|l|l|l|l|}
\hline Prior & \multicolumn{5}{|c|}{$\alpha=2, \beta=5$} & \multicolumn{4}{c|}{$\alpha=2, \beta=7$} \\
\hline $\mathrm{n}$ & 30 & 50 & 70 & 100 & 30 & 50 & 70 & 100 \\
\hline UP & 2.0327 & 2.0197 & 2.0245 & 2.0297 & 2.0268 & 2.0006 & 2.8370 & 2.0423 \\
& $(0.0284)$ & $(0.0201)$ & $(0.0141)$ & $(0.0283)$ & $(0.0202)$ & $(0.0384)$ & $(0.0814)$ & $(0.0595)$ \\
\hline JP & 2.5031 & 2.4997 & 2.4997 & 2.5009 & 2.2509 & 2.2488 & 2.2511 & 2.2561 \\
& $(0.8592)$ & $(0.7341)$ & $(0.6608)$ & $(0.5990)$ & $(0.1406)$ & $(0.7734)$ & $(0.1992)$ & $(0.8355)$ \\
\hline & \multicolumn{6}{|c|}{$\alpha=4, \beta=5$} \\
\hline UP & 4.4740 & 4.6367 & 4.0937 & 3.8370 & 4.0423 & 4.5385 & 4.0327 & 3.0197 \\
& $(0.5020)$ & $(0.0306)$ & $(0.0720)$ & $(0.0814)$ & $(0.0595)$ & $(0.0876)$ & $(0.0284)$ & $(0.0201)$ \\
\hline JP & 4.5031 & 4.4997 & 4.4997 & 4.5009 & 4.2509 & 4.2488 & 4.2511 & 4.2561 \\
& $(0.8592)$ & $(0.7341)$ & $(0.6608)$ & $(0.5990)$ & $(0.1406)$ & $(0.7734)$ & $(0.1992)$ & $(0.8355)$ \\
\hline
\end{tabular}




\section{Conclusion}

To study behavior of scale parameter of Log logistic distribution, Simulation study is used. It is concluded that Bayes estimators approaches to their true value and posterior risks is decreases by increasing sample size. Jeffery prior is performing better than uniform prior as its posterior risk is minimum. Precautionary loss function is performing better as its posterior risk is least among all assumed loss functions. So Jeffery with precautionary loss function provides minimum posterior risks as compared to other loss functions and priors.

The study can be further extended by using others loss functions and prior distributions.

\section{References}

[1] Singh, VP and Guo, H and Yu, FX, "Parameter estimation for 3-parameter log-logistic distribution (LLD3) by Pome.", Stochastic Hydrology and Hydraulics, Vol(7), (1993), pp.163-177

[2] Seefeldt, Steven S and Jensen, Jens Erik and Fuerst, E Patrick., " Log-logistic analysis of herbicide dose-response relationships.", Weed technology,(1995), pp.218-227.

[3] Rowinski, Pawel M and Strupczewski, Witold G and Singh, Vijay P, "A note on the applicability of log-Gumbel and log-logistic probability distributions in hydrological analyses: I. Known pdf." Hydrological sciences journal, Vol(47), (2002), pp.107-122

[4] elsherpieny2013discriminating., "Discriminating between Weibull and log-logistic distributions" International Journal of Innovative Research in Science, Engineering and Technology, Vol(2), (2013), pp.3358-3371

[5] Akhtar, Md Tanwir and Khan, Athar Ali., "Log-logistic Distribution as a Reliability Model: A Bayesian Analysis." American Journal of Mathematics and Statistics, Vol(4), (2014), pp.162-170

[6] Kus, Coskun and Kaya, Mehmet Fedai., "Estimation of Parameters of the Loglogistic Distribution based on Progressive Censoring using the EM Algorithm", Hacettepe Journal of Mathematics and Statistics, $\operatorname{Vol}(35),(2006)$ 